\title{
Fragile Histidine Triad Protein, WW Domain-containing Oxidoreductase Protein Wwox, and Activator Protein $2 \gamma$ Expression Levels Correlate With Basal Phenotype in Breast Cancer
}

Gulnur Guler, MD¹, Kay Huebner, $\mathrm{PhD}^{2}$, Cigdem Himmetoglu, MD¹, Rafael E. Jimenez, MD ${ }^{3}$, Stefan Costinean, $M^{2}$, Stefano Volinia, $\mathrm{PhD}^{2}$, Robert T. Pilarski, $\mathrm{MS}^{4}$, Mutlu Hayran, MD ${ }^{5}$, and Charles L. Shapiro, $\mathrm{MD}^{6}$

BACKGROUND: The expression of fragile histidine triad protein (Fhit) and WW domain-containing oxidoreductase protein (Wwox), tumor suppressors that are encoded by fragile (FRA) loci FRA3B and FRA16D, are lost concordantly in breast cancers. In the current study, the authors examined correlations among Fhit, Wwox, the activator protein 2 transcription factors AP2 $\alpha$ and AP2 $\gamma$, cytokeratins 5 and 6 (CK5/6), epidermal growth factor receptor (EGFR), estrogen receptor (ER), progesterone receptor (PR), and human epidermal growth factor receptor 2 (HER-2) and their associations with breast cancer phenotypes. METHODS: Tissue microarrays constructed from 837 breast cancer blocks were immunostained. Expression in $>10 \%$ of tumor cells was considered positive for cytoplasmic CK5/6, membranous EGFR, and nuclear AP2 $\alpha$ and AP2 $\gamma$. Cytoplasmic Fhit and Wwox staining was scored according to staining intensity. ER, PR, and HER-2 status of tumors was derived from records. Correlations among immunohistochemical markers and tumor subtypes were assessed by univariate and multivariate statistical methods. RESULTS: Triple-negative tumors had more frequent expression of EGFR, CK5/6 $(P<.001)$, and AP2 $\gamma(P=.003)$ and more frequent loss of Fhit and Wwox $(P<.001)$, and an inverse correlation was observed between Fhit, Wwox expression and EGFR, ER, and PR expression $(P<.001)$. Reduced Fhit expression was more common in HER-2-positive and AP2 $\gamma$-positive cases $(P<.001$ and $P=.002$, respectively). There was a direct correlation noted between Fhit and Wwox $(P<.001)$ and a borderline positive relation between AP2 $\alpha$ and AP2 $\gamma(P=.054)$. CONCLUSIONS: The results from this investigation suggested that reduced expression levels of Fhit, Wwox, and nuclear AP2 $\gamma$ have roles in the pathogenesis of basal-like differentiation in breast cancer. Alteration in

Corresponding author: Kay Huebner, PhD, Department of Molecular Virology, Immunology and Medical Genetics, Ohio State University Comprehensive Cancer Center, Biomedical Research Tower, Room 916, 460 W. 12th Avenue, Columbus, OH 43210; Fax: (614) 688-8675; kay.huebner@osumc.edu

${ }^{1}$ Department of Pathology, Hacettepe University, Ankara, Turkey; ${ }^{2}$ Department of Molecular Virology, Immunology, and Medical Genetics, Ohio State University Medical Center, Columbus, Ohio; ${ }^{3}$ Department of Pathology, Ohio State University Medical Center, Columbus, Ohio; ${ }^{4}$ Division of Clinical Genetics, Ohio State University Medical Center, Columbus, Ohio; ${ }^{5}$ Institute of Oncology and Preventive Oncology, Hacettepe University, Ankara, Turkey; ${ }^{6}$ Division of Hematology and Oncology, Department of Internal Medicine, Ohio State University Medical Center, Columbus, Ohio

Rafel Jimenez's current address: Division of Anatomic Pathology, Mayo Clinic, Rochester, Minnesota

The first 2 authors contributed equally to this article.

We gratefully acknowledge the outstanding technical services of individuals in the Pathology Core Facility of the Ohio State University, especially Susie Jones.

Received: July 9, 2008; Revised: September 5, 2008; Accepted: September 10, 2008

Published online: January 6, 2009, (c) 2009 American Cancer Society

DOI: 10.1002/cncr.24103, www.interscience.wiley.com 
the expression of fragile site genes occurs in most of these cancers and may contribute to defects in DNA repair, as observed in breast cancer 1 (BRCA1)-deficient cancers. Thus, DNA damage response checkpoint proteins may be targets for treatment. Cancer 2009;115:899-908. (C) 2009 American Cancer Society.

KEY WORDS: breast cancer subtypes, triple-negative breast cancers, common fragile sites, fragile histidine triad protein, WW domain-containing protein, activator protein 2 transcription factors.

Common chromosomal fragile sites are preferential targets of replication stress in preneoplastic lesions, resulting in deletions that involve the fragile genes encoded at these conserved chromosome regions. ${ }^{1,2}$ It has been suggested that damage to fragile sites, with lost function of genes located at these sites, may play a significant role in carcinogenesis. ${ }^{3}$ Reduced expression of the fragile tumor suppressors, fragile histidine triad protein (Fhit) and the WW domain-containing oxidoreductase protein Wwox, which are encoded by fragile (FRA) loci $F R A 3 B$ and FRA16D at chromosomes 3p14.2 and 16q23.3, are lost concordantly in approximately $66 \%$ of breast cancers. ${ }^{4}$ Expression levels of Fhit and Wwox have been associated with important aspects of breast cancer initiation, response to therapy, and clinicopathologic features. ${ }^{4-7}$ Their coordinated loss also has been demonstrated in other types of cancers and cells. ${ }^{8,9}$

Our group and others have reported that Fhit and Wwox loss is more common in breast cancers that are negative for estrogen receptor (ER) and progesterone receptor $(\mathrm{PR})^{4,7}$ and that Fhit loss is significantly more frequent in human epidermal growth factor receptor 2 (HER-2)-positive ductal carcinoma in situ (DCIS). ${ }^{5}$ In another study, we also observed that expression levels of Wwox, the Wwox-interacting activator protein $2 \gamma$ (AP2 $\gamma$ ), ErbB $4,{ }^{10,11}$ and the AP2 $\gamma$ transcriptional target protein HER-2 ${ }^{12}$ were correlated with tamoxifen resistance in ER-positive, invasive breast cancers. ${ }^{6}$ Lost or reduced expression of Wwox, high-level expression of AP $2 \gamma$ and HER-2, and ErbB4 loss were identified as independent markers of tamoxifen resistance. Reduced Wwox expression was better than PR expression for predicting resistance, and nuclear AP $2 \gamma$ expression was better than HER2 expression. ${ }^{6}$

The recently described, molecular-based classification of breast cancers has defined 3 groups with negative ER status: basal-like, HER-2 overexpressing, and normal breast-like cancers. ${ }^{13,14}$ Understanding the pathogenic mechanisms of the basal-like and triple-negative (ie, negative for ER, PR, and HER-2) breast cancer subtypes is particularly important because, contrary to disease that is positive for hormone receptors or HER-2, no targeted therapies for these tumors have been developed to date, and chemotherapy remains the only therapeutic option for these patients. ${ }^{15-17}$ Both triple-negative and basal-like cancers (which mostly have a triple-negative phenotype) preferentially affect young women and women of African origin, they usually are of high histologic grade, and they exhibit more aggressive clinical behavior. ${ }^{16}$ Overlap between the biologic and clinical characteristics of sporadic triple-negative/basal-like cancers and cancers in breast cancer gene BRCA1 mutation carriers has been demonstrated, ${ }^{18,19}$ and the basal cell-like molecular breast cancer subtype is an independent predictor of distant metastasis in African-American women. ${ }^{17}$

The current study was designed to determine whether alterations in the expression of genes at fragile sites contribute preferentially to the pathogenesis of certain subtypes of breast cancer. We determined the expression levels for tumor suppressor genes FHIT and WWOX (located at the 2 most active fragile sites of human genome) and the transcription factors AP2 $\alpha$ and AP $2 \gamma$ (which interact physically with $\mathrm{W}_{\text {wox }}$ ) in a large cohort of breast cancers that we attempted to classify molecularly according to their expression of the hormone receptors ER and PR, HER-2, cytokeratins 5 and 6 (CK5/6), and epidermal growth factor receptor (EGFR), which are markers of basal-like differentiation. ${ }^{20,21}$

\section{MATERIALS AND METHODS}

\section{Construction of Tissue Microarrays}

This study had the approval of the Ohio State University Institutional Review Board for studies of human subjects. Tissue microarrays (TMAs) were prepared from cores of the tissue blocks from breast cancers in 837 women from 
Table 1. Primary Antisera and Detection Kits Used in Immunohistochemical Studies

\begin{tabular}{|c|c|c|c|c|}
\hline $\begin{array}{l}\text { Primary } \\
\text { Antiserum }\end{array}$ & Source & Description & Dilution & Detection Kit \\
\hline Fhit & Huebner Laboratory* & Rabbit polyclonal & $1 / 5000$ & $\begin{array}{l}\text { Vectastain Elite kit (Vector Laboratories, } \\
\text { Burlingame, Calif) }\end{array}$ \\
\hline Wwox & Huebner Laboratory* & Rabbit polyclonal & $1 / 7000$ & Vectastain Elite kit (Vector Laboratories) \\
\hline $\mathrm{AP} 2 \gamma$ & $\begin{array}{l}\text { Santa Cruz Biotechnology } \\
\text { (Santa Cruz, Calif) }\end{array}$ & Mouse monoclonal (6E4/4) & $1 / 75$ & Universal LSAB+ (Dako, Carpinteria, Calif) \\
\hline $\mathrm{AP} 2 \alpha$ & Santa Cruz Biotechnology & Mouse monoclonal (3B5) & $1 / 75$ & Universal LSAB + (Dako) \\
\hline CK5/6 & Dako & Mouse monoclonal (D5/16 B4) & $1 / 50$ & Universal LSAB+ (Dako) \\
\hline EGFR & PharmDx Kit (Dako) & Mouse monoclonal (2-18C9) & Neat & Envision Plus, included in kit (Dako) \\
\hline
\end{tabular}

Fhit indicates fragile histidine triad protein; Wwox, WW domain-containing oxidoreductase protein; AP2 $\gamma$, activator protein $2 \gamma$; AP2 $\alpha$, activator protein $2 \alpha$; CK5/ 6, cytokeratins 5/6; EGFR; epidermal growth factor receptor.

*The features of the antisera have been described previously in detail (see Guler 2005, $2007^{5,6}$ ).

the 4 major hospitals in Columbus, Ohio. The clinical data on patients; histologic tumor type and grade (according to the modified Bloom-Richardson system); and ER, PR, and HER-2 status of tumors were obtained from clinical records and pathology reports. ER and PR status was evaluated clinically by immunohistochemistry (IHC), and samples that had nuclear staining for either receptor in $>1 \%$ of cells were regarded as positive. HER-2 status was evaluated clinically by using a standard combined IHC plus fluorescence in situ hybridization (FISH) approach. All samples that had intense, complete, membranous staining in $>10 \%$ of invasive neoplastic cells determined by IHC or the presence of HER-2 gene amplification determined by FISH were considered HER-2 positive.

\section{Immunohistochemical Analysis}

Fhit, Wwox, CK5/6, EGFR, AP2 $\alpha$, and AP2 $\gamma$ immunostaining was performed on TMA sections. The specific antisera, staining methods, detection kits, and controls that we used are listed in Table 1. Antigen retrieval was the same for all antisera with the exception of EGFR and CK5/6; sections were boiled in citrate buffer ( $\mathrm{pH}$ 6.0) in a vegetable steamer for 25 minutes and cooled in solution for 15 minutes. For EGFR antigen retrieval, we used Proteinase $\mathrm{K}$ from the PharmDx kit. Antigen retrieval for CK5/6 was in ethylene diamine tetraacetic acid buffer $(\mathrm{pH} 9.0)$ in a vegetable steamer for 25 minutes with cooling in solution for 15 minutes. Details of IHC staining methods have been described previously. ${ }^{4,5}$

The expression of Fhit, Wwox, and CK5/6 was cytoplasmic, the expression of AP2 $\alpha$ and AP $2 \gamma$ was nuclear, and the expression of EGFR was both cytoplasmic and membranous. Cytoplasmic expression in $\geq 10 \%$ of tumor cells for CK5/6, membranous staining in $\geq 10 \%$ of tumor cells for EGFR, and nuclear staining in $\geq 10 \%$ of tumor cells for AP $2 \alpha$ and AP $2 \gamma$ were accepted as positive. Fhit staining and Wwox staining in tumors were scored according to staining intensity as follows: absent staining, highly reduced staining, reduced staining, or strong expression. Three pathologists scored the slides and were blinded to the subtype of breast cancer.

The 837 breast cancers were divided into subtypes of breast cancer as defined by their IHC profiles using the designated stains. ${ }^{15,20,21}$ These included luminal A (positive for ER and/or PR and negative for HER-2), luminal $\mathrm{B}$ (positive for ER and/or PR and positive for HER-2), HER-2 overexpressing (negative for ER and PR and positive for HER-2), basal-like triple negative (negative for ER, PR, and HER-2 and positive for CK5/6 and/or EGFR), and other triple-negative (negative for ER, PR, HER-2, CK5/6, and EGFR).

\section{Statistical Analysis}

Associations among specific markers and specific clinical features were analyzed using the chi-square test or the Fisher exact test, as appropriate, for univariate analyses and multiple logistic regression for multivariate analysis, as described previously. ${ }^{6}$ Pairwise correlations of biomarkers were assessed using the Spearman correlation test. $P$ values of .05 were considered statistically significant. 


\section{RESULTS}

\section{Characteristics of the Breast Cancers}

The demographic characteristics and the various IHC results are described in Table 2 . The median patient age was 55 years (range, 20-93 years), and 100 patients (12\%) were aged $<40$ years. Seven hundred fifty-nine patients (90\%) were white, 59 patients (7\%) were black, and the remaining patients were of other races/ethinicities. These characteristics reflected the catchment areas of the 4 contributing hospitals. Seven hundred and forty-nine patients $(89.5 \%)$ had invasive ductal carcinoma, not otherwise specified; 34 patients $(4.1 \%)$ had invasive lobular carcinoma, 24 patients (2.9\%) had mixed ductal and lobular carcinoma, 12 patients $(1.4 \%)$ had mucinous carcinoma, and 18 patients $(2.1 \%)$ had other specific types of invasive breast cancer, such as medullary, tubular, or papillary carcinoma.

\section{Expression of Fhit and Wwox Assessed by Immunohistochemistry Analyses on TMAs}

There was a significant, positive relation between Fhit expression and Wwox expression $(P=.024)$. In univariate analysis, when we compared Fhit expression with prognostic markers (Table 2), Fhit loss was more frequent in infiltrating ductal carcinoma; $65.7 \%$ of ductal carcinomas had highly reduced or negative Fhit expression, whereas $54.3 \%$ of infiltrating lobular carcinomas and $33.3 \%$ of other types of invasive tumors had highly reduced or negative Fhit expression $(P=.01)$. Fhit expression also was related to histologic tumor grade; $78.3 \%$ of grade 3 tumors, $59.4 \%$ of grade 2 tumors, and $36.7 \%$ of grade 1 tumors had lost or highly reduced Fhit expression $(P<$ $.001)$. Fhit expression and patient age were related significantly; lost or highly reduced Fhit expression was observed in $84.1 \%$ of tumors among patients aged $\leq 40$ years, in $70.9 \%$ of tumors among patients ages 41 to 50 years, and in $59 \%$ of tumors among patients aged $>50$ years $(P<$ $.001)$. Very low Fhit expression was more frequent in tumors from African-American women (84.6\%) than in tumors from white women $(63 \% ; P=.002)$.

Wwox expression scores also were compared with the same prognostic features (Table 2): Wwox expression was related to tumor type; $78.5 \%$ of infiltrating ductal carcinomas, $64.4 \%$ of infiltrating lobular carcinomas, and
$78.3 \%$ of other types of invasive tumors had highly reduced or no Wwox expression $(P=.01)$. Wwox expression also was associated with histologic grade; $84.6 \%$ of grade 3 tumors, $72.6 \%$ of grade 2 tumors, and $72 \%$ of grade 1 tumors had highly reduced or absent Wwox expression $(P<.001)$. Wwox expression was not found to be related to patient age. Highly reduced or absent Wwox expression was more frequent in tumors from AfricanAmerican women $(88.1 \%)$ than in tumors from white women $(76 \% ; P=.034)$. The clinicopathologic features of the tumors and their associations with Fhit and Wwox expression are provided in Table 2.

\section{Associations Among Breast Cancer Marker Proteins and Clinicopathologic Features}

Fhit expression scores were assessed in association with the expression of other biomarkers (for examples of IHC stains, see Fig. 1). There was a strong, direct correlation between Fhit expression and the expression of both ER and PR $(P<.001$ for both); whereas an inverse correlation was observed between Fhit expression and the expression of HER-2 $(P<.001)$, EGFR $(P<.001)$, CK5/6 $(P=.018)$, and AP2 $\gamma(P=.002)$ (Table 2$)$.

Wwox expression also was related highly and positively with the expression of both ER $(P<.001)$ and PR $(P=.001)$, an inverse correlation was observed between Wwox expression and the expression of EGFR $(P<$ $.001)$, and a borderline inverse correlation was observed between Wwox expression and the expression if CK5/6 ( $P$ $=.071)$. There was no association between Wwox expression and the expression of HER-2 $(P=.204)$, AP $2 \alpha$ $(P=.607)$, or $\mathrm{AP} 2 \gamma(P=.171)$ in univariate analyses.

We characterized the basal-like phenotype by using 5 biomarkers that included triple negativity for ER, PR, HER-2 and positive expression of CK5/6 and/or EGFR. ${ }^{13,14}$ According to this classification, 89 tumors (10.7\%) exhibited basal-like differentiation (Table 3). Next, we assessed the relation of the basal-like phenotype to expression scores for Fhit, Wwox, AP $2 \gamma$, and AP $2 \alpha$ and observed a highly significant association of the basallike phenotype with very low expression of Fhit and Wwox $(P<00.1)$ and high expression of AP2 $\gamma(P<.001)$ (Table 3; for examples, see Fig. 1).

When we examined correlations between the expression of biomarkers and the expression of Fhit and Wwox 
Table 2. Clinicopathologic Features of Tumors, Biomarker Expression Levels, and Associations With Expression of the Fragile Histidine Triad Protein and the WW Domain-containing Oxidoreductase Protein

\section{Fhit Expression}

No. of Patients (\%)

\begin{tabular}{|c|c|c|c|c|c|c|}
\hline Feature & $\begin{array}{l}\text { Lost or } \\
\text { Highly } \\
\text { Reduced }\end{array}$ & $\begin{array}{l}\text { Strong or } \\
\text { Moderately } \\
\text { Reduced }\end{array}$ & $P$ & $\begin{array}{l}\text { Lost or } \\
\text { Highly } \\
\text { Reduced }\end{array}$ & $\begin{array}{l}\text { Strong or } \\
\text { Moderately } \\
\text { Reduced }\end{array}$ & $P$ \\
\hline \multicolumn{7}{|l|}{ Age, y } \\
\hline$\leq 40$ & 69 (84.1) & $13(15.9)$ & $<.001$ & 79 (79.8) & $20(20.2)$ & .512 \\
\hline $41-50$ & 117 (70.9) & $48(29.1)$ & - & $155(79.1)$ & $41(20.9)$ & - \\
\hline$>50$ & $265(59)$ & $184(41)$ & - & $398(75.8)$ & $127(24.2)$ & - \\
\hline \multicolumn{7}{|l|}{ Histologic type } \\
\hline Invasive ductal, NOS & $414(65.7)$ & $216(34.3)$ & .010 & 579 (78.5) & $159(21.5)$ & .036 \\
\hline Invasive lobular & $25(54.3)$ & $21(45.7)$ & - & $38(64.4)$ & $21(35.6)$ & - \\
\hline Other invasive & $6(54.5)$ & $5(45.5)$ & - & $7(58.3)$ & $5(41.7)$ & - \\
\hline \multicolumn{7}{|l|}{ Histologic grade } \\
\hline 1 & $33(36.7)$ & $57(63.3)$ & $<.001$ & $85(72)$ & $33(28)$ & $<.001$ \\
\hline 2 & $155(59.4)$ & $106(40.6)$ & - & 225 (72.6) & $85(27.4)$ & - \\
\hline 3 & $241(78.2)$ & $67(21.8)$ & - & $291(84.6)$ & $53(15.4)$ & - \\
\hline \multicolumn{7}{|l|}{ Race } \\
\hline Caucasian & $402(63)$ & $236(37)$ & .001 & $574(76)$ & $181(24)$ & .036 \\
\hline African American & $44(84.6)$ & $8(15.4)$ & - & $52(88.1)$ & $7(11.9)$ & - \\
\hline \multicolumn{7}{|l|}{ ER status } \\
\hline Positive & $297(57.9)$ & $261(42.1)$ & $<.001$ & $443(73)$ & $164(27)$ & $<.001$ \\
\hline Negative & $154(84.2)$ & $29(15.8)$ & - & $187(88.2)$ & $25(11.8)$ & - \\
\hline \multicolumn{7}{|l|}{ PR status } \\
\hline Positive & $237(55.4)$ & $191(44.6)$ & $<.001$ & $373(73)$ & $138(27)$ & .001 \\
\hline Negative & 214 (79.9) & $54(20.1)$ & - & $258(83.5)$ & $51(16.5)$ & - \\
\hline \multicolumn{7}{|l|}{ HER-2 status } \\
\hline Positive & $121(77.1)$ & 36 (22.9) & $<.001$ & $136(73.5)$ & 49 (26.5) & .233 \\
\hline Negative & $324(61.1)$ & 206 (38.9) & - & 489 (78) & 138 (22.8) & - \\
\hline \multicolumn{7}{|l|}{ CK5/6 } \\
\hline Positive & 95 (74.2) & $33(25.8)$ & .021 & $110(82.1)$ & $24(17.9)$ & .071 \\
\hline Negative & $309(63.1)$ & $181(36.9)$ & - & 399 (74.6) & $136(25.4)$ & - \\
\hline \multicolumn{7}{|l|}{ EGFR } \\
\hline Positive & $108(85)$ & 19 (15) & $<.001$ & $123(91.1)$ & $12(8.9)$ & $<.001$ \\
\hline Negative & $312(59.8)$ & $210(40.2)$ & - & $430(73)$ & $155(27)$ & - \\
\hline \multicolumn{7}{|l|}{ AP2 $\alpha$} \\
\hline Positive & $368(64.7)$ & 201 (35.3) & .584 & 437 (73.8) & 155 (26.2) & .607 \\
\hline Negative & 55 (70.5) & $23(29.5)$ & - & 70 (76.9) & $21(23.1)$ & - \\
\hline \multicolumn{7}{|l|}{ AP2 $\gamma$} \\
\hline Positive & $301(71)$ & $123(29)$ & .002 & $322(73.2)$ & $18(26.8)$ & .171 \\
\hline Negative & $135(58.7)$ & $95(4.3)$ & - & $196(78.1)$ & 55 (21.9) & - \\
\hline
\end{tabular}

Fhit indicates fragile histidine triad protein; Wwox, WW domain-containing oxidoreductase protein; NOS, not otherwise specified; ER, estrogen receptor; PR, progesterone receptor; HER-2, epidermal growth factor receptor family member 2; CK5/6, cytokeratins 5 and 6; EGFR, epidermal growth factor receptor; AP $2 \alpha$; activator protein $2 \alpha$; AP $2 \gamma$, activator protein $2 \gamma$.

in a regression analysis, we observed that the 3 markers most closely associated with Fhit were ER, EGFR, and HER-2; whereas PR, EGFR, and HER-2 were associated most closely with Wwox expression (Tables 4 and 5). We divided the tumors into 5 groups according to expression patterns of these 5 biomarkers with the objective of finding tumors that matched the recently described genetic classification $^{11,12}$ : luminal A (positive for ER and/or PR and negative for HER-2), luminal B (positive for ER and/ or PR and positive for HER-2), HER-2 overexpressing 


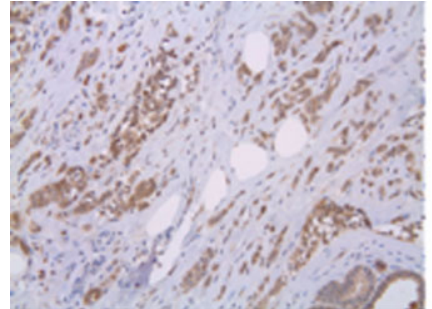

Fhit+ tumor

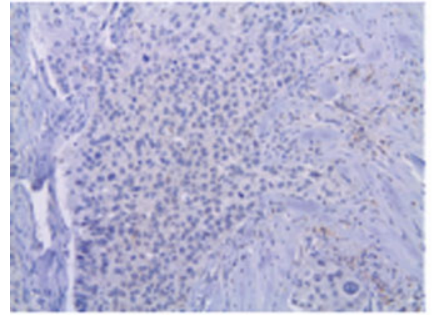

827 Fhit

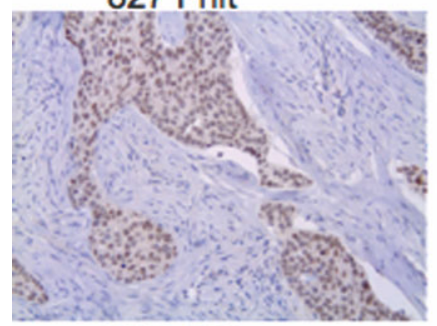

827 AP2 $\alpha$

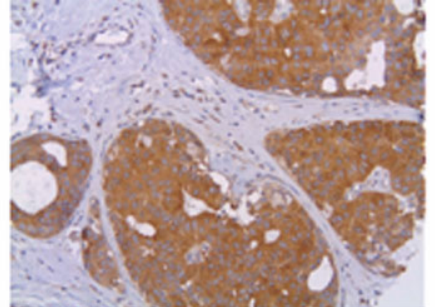

Wwox+ tumor

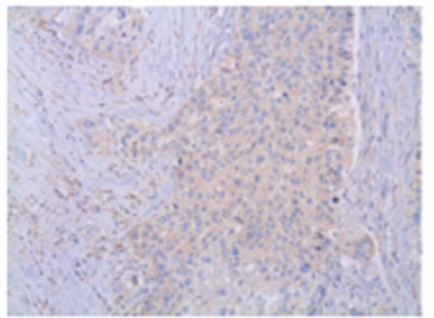

827 Wwox

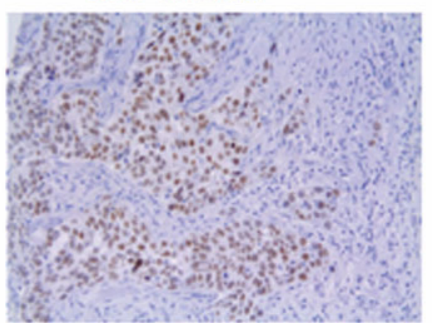

827 AP2 $\gamma$

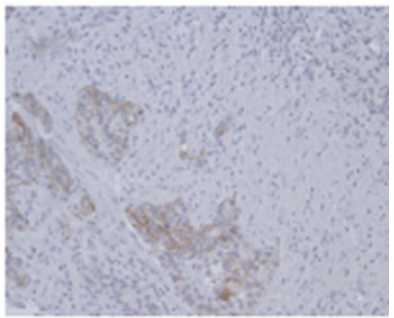

827 CK5,6

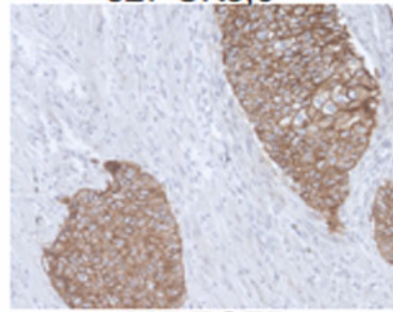

827 EGFR

FIGURE 1. Immunohistochemical staining of protein markers in a triple-negative breast cancer. In the top 2 photomicrographs, strong expression of fragile histidine triad protein (Fhit) and the WW domain-containing oxidoreductase protein Wwox is observed, in contrast to the negative and very low expression of Fhit and Wwox, respectively, in triple-negative Tumor 827. Tumor 827 also demonstrated moderate expression of cytokeratins 5 and 6 (CK5.6), moderate nuclear expression of activator proteins $2 \alpha(\mathrm{AP} 2 \alpha)$ and $2 \gamma(\mathrm{AP} 2 \gamma)$, and strong membranous expression of epidermal growth factor receptor (EGFR) (original magnification $\times 200$ in all photomicrographs).

(negative for ER and PR and positive for HER-2), basallike triple negative (negative for ER, PR, and HER-2 and positive for CK5/6 and/or EGFR), and other (triple negative but not positive for CK5/6 or EGFR) (Table 3).

Expression patterns of the protein biomarkers within specific subtypes were assessed, and highly significant associations were observed for Fhit, Wwox, and AP2 $\gamma(P<.001$ for all). Lost or highly reduced Fhit expression was observed in $88.1 \%$ of HER-2 overexpressing tumors and in $84.5 \%$ of basal-like tumors; whereas $55.5 \%$ of luminal A tumors, $70.4 \%$ of luminal B tumors, and $75 \%$ of triple-negative tumors with other than basallike phenotype had very low Fhit expression. When we assessed the association of different tumor subtypes in relation to Wwox expression scores, absent or highly reduced Wwox expression occurred in $96.6 \%$ of basal-like tumors, in $90 \%$ of triple-negative tumors that were other than basal-like, in $80 \%$ of HER-2 overexpressing tumors, in $74.2 \%$ of luminal A tumors, and in $69.6 \%$ of luminal B tumors (see Table 3).

\section{Coordinated Loss of Fhit and Wwox Expression}

Highly reduced or lost expression of both Fhit and Wwox was observed in 342 tumors (49\%). It is likely that the effect of reduced Fhit and Wwox expression is not very different from complete loss, because it is known that Fhit and Wwox are haploinsufficient tumor suppressors (ie, gene knockout animals with loss of 1 or both copies are equally highly susceptible to tumor induction). ${ }^{22-24}$ The basal-like phenotype was observed in $20.7 \%$ of these 
Table 3. The Relation of Genetically Defined Breast Cancer Subtypes With Fragile Histidine Triad Protein and WW Domain-containing Oxidoreductase Protein Expression

\begin{tabular}{|c|c|c|c|c|c|c|}
\hline \multirow[b]{2}{*}{ Subtype } & \multicolumn{3}{|c|}{ Fhit Expression } & \multicolumn{2}{|c|}{ Wwox Expression } & \multirow[b]{2}{*}{$P$} \\
\hline & $\begin{array}{l}\text { Lost or } \\
\text { Highly } \\
\text { Reduced }\end{array}$ & $\begin{array}{l}\text { Strong or } \\
\text { Moderately } \\
\text { Reduced }\end{array}$ & $P$ & $\begin{array}{l}\text { Lost or } \\
\text { Highly } \\
\text { Reduced }\end{array}$ & $\begin{array}{l}\text { Strong or } \\
\text { Moderately } \\
\text { Reduced }\end{array}$ & \\
\hline \multicolumn{7}{|l|}{ Triple negative } \\
\hline Positive & 95 (81.9) & 21 (18.1) & $<.001$ & $121(92.4)$ & $10(7.6)$ & $<.001$ \\
\hline Negative & $361(61.7)$ & $224(38.3)$ & - & $514(74.2)$ & $179(25.8)$ & - \\
\hline \multicolumn{7}{|l|}{ Basal-like phenotype } \\
\hline Positive & 71 (84.5) & 13 (15.5) & $<.001$ & 86 (96.6) & $3(3.4)$ & $<.001$ \\
\hline Negative & $380(62.1)$ & $232(37.9)$ & - & $545(74.6)$ & $186(25.4)$ & - \\
\hline \multicolumn{7}{|l|}{ Genetic classification } \\
\hline Luminal A & $229(55.3)$ & $185(44.7)$ & $<.001$ & $368(74.2)$ & $128(25.8)$ & $<.001$ \\
\hline Luminal B & $69(70.4)$ & 29 (29.6) & - & $80(69.6)$ & $35(30.4)$ & - \\
\hline HER-2 overexpression & $52(88.1)$ & $7(11.9)$ & - & $56(80.6)$ & $14(20)$ & - \\
\hline Basal-like & 71 (84.5) & 13 (15.5) & - & 86 (96.6) & $3(3.4)$ & - \\
\hline Triple negative-other & $15(75)$ & $5(25)$ & - & $18(90)$ & $2(10)$ & - \\
\hline
\end{tabular}

Fhit indicates fragile histidine triad protein; Wwox, WW domain-containing oxidoreductase protein; HER-2, human epidermal growth factor receptor 2.

Table 4. Multivariate Logistic Regression Analysis of Biomarkers Associated With the Fragile Histidine Triad Protein

\begin{tabular}{lllll} 
Biomarkers & $\boldsymbol{P}$ & OR & \multicolumn{2}{c}{$\mathbf{9 5 \%} \mathbf{C l}$ for OR } \\
& & & Lower & Upper \\
& & & & \\
EGFR & .013 & 3.3 & 1.18 & 3.95 \\
ER & .0001 & 14.6 & 1.63 & 4.20 \\
HER-2 & .019 & 30.8 & 1.1 & 3.03 \\
\hline
\end{tabular}

OR indicates odds ratio; 95\% Cl, 95\% confidence interval; EGFR, epidermal growth factor receptor; ER, estrogen receptor; HER-2, human epidermal growth factor receptor family member 2 .

tumors, which was significantly more compared with tumors that had high or moderately reduced Fhit and/or Wwox expression $(3.9 \% ; P<.001)$. In other words, $83.3 \%$ of basal-like tumors had very low or lost expression of both Fhit and Wwox; and $31.6 \%$ of these basal-like tumors (vs 7.9\% of Fhit- and/or Wwox-positive tumors; $P<.001)$ expressed EGFR. CK5/6 expression was observed in $25.9 \%$ of Fhit- and Wwox-negative tumors and in $15.6 \%$ of Fhit- and/or Wwox-positive tumors $(P=.002)$. HER-2 status did not differ significantly between the 2 groups $(P=.174)$.

\section{DISCUSSION}

Our previous studies on the role of fragile genes in breast cancer demonstrated that Fhit and Wwox expression is
Table 5. Multivariate Logistic Regression Analysis of Biomarkers Associated With the WW Domain-containing Oxidoreductase Protein

\begin{tabular}{llllc} 
Biomarkers & $\boldsymbol{P}$ & OR & \multicolumn{2}{c}{$\mathbf{9 5 \%}$ Cl for OR } \\
& & & Lower & Upper \\
& & & & \\
EGFR & .008 & 2.64 & 1.29 & 5.41 \\
PR & .004 & 2.43 & 1.33 & 4.44 \\
HER-2 & .026 & 1.69 & 1.06 & 2.68 \\
& & & & \\
\hline
\end{tabular}

OR indicates odds ratio; 95\% Cl, 95\% confidence interval; EGFR, epidermal growth factor receptor; PR, progesterone receptor; HER-2, human epidermal growth factor receptor family member 2 .

reduced or lost in up to $60 \%$ of invasive breast cancers and pure DCIS, and up to $50 \%$ of expression loss is observed in adjacent normal cell areas of breast cancer sections. ${ }^{4,5}$ In those studies, in which Fhit and Wwox expression was examined in invasive breast tumors, ${ }^{4}$ in tamoxifen-sensitive and tamoxiven-resistant breast cancers, ${ }^{6}$ in pure DCIS, in DCIS adjacent to invasive tumors, and in normal tissues around invasive or in situ breast cancers, ${ }^{5}$ Fhit expression and Wwox expression were correlated positively in all tissue compartments. Loss of Fhit and Wwox was more common in ER- and PR-negative breast tumors. ${ }^{1}$ Nunez et $\mathrm{al}^{7}$ confirmed this relation of ER and Wwox in breast cancer. The concordant inactivation of Fhit and Wwox is an early event in breast carcinogenesis and is especially common in ER- and PR-negative breast tumors. 
Recently described breast cancer subtypes, identified through gene expression profiles or signatures, have defined 5 breast cancer groups with different prognostic features: luminal A, luminal B, HER-2 overexpressing, basal-like, and normal breast-like. ${ }^{13,14}$ Basal-like breast cancers are triple-negative tumors (negative for ER, PR, and HER-2) and, currently, the best method for identifying basal-like tumors is by routine IHC that is positive for CK5/6 and/or EGFR and negative for ER, PR, and HER-2. ${ }^{20,21}$ It has been reported that this 5-marker panel has $100 \%$ specificity and $76 \%$ sensitivity ${ }^{20}$ in identifying basal-like cancers. Basal-like cancers, so named because they express genes that are present in normal basal/myoepithelial cells of the breast, account for up to $15 \%$ of all breast cancers but accounted for $>50 \%$ of breast cancers in some cohorts of patients in Africa. ${ }^{17}$ Mutant BRCA1-associated breast cancers have basal-like features, and BRCAI inactivation is a common event in sporadic basal-like breast tumors. ${ }^{18,19}$ In various studies of sporadic breast cancers, $\geq 40 \%$ of tumors were moderately to strongly positive for Fhit, whereas only $9 \%$ of BRCA1 mutant tumors were positive for Fhit, suggesting that the $B R C A 1$ pathway is important in protecting the FRA3B/FHIT locus from damage ${ }^{25}$; inactivation of the $B R C A 1$ pathway in basal/triple-negative cancers may contribute to loss of expression of Fhit in these cancers or, possibly, vice versa.

The objective of this study was to determine the relation of concordant Fhit and Wwox loss in the carcinogenesis of currently described breast cancer subtypes. This study revealed strong correlations between the triple-negative phenotype or the basal-like phenotype (triple negative and positive for CK5/6 and/or EGFR) and reduced Fhit and Wwox expression. The tumors with concordant loss of Fhit and Wwox were much more likely to be basal-like tumors than the tumors that strongly expressed Fhit or Wwox. The results indicate that the inactivation of fragile site genes is associated highly with and may play a role in the formation of basal-like breast cancers. The molecular events involved in this process need to be investigated in in vivo experiments to help to identify new targets for treatment.

An interesting area of investigation would be the relation between basal-like breast cancers and activation of the DNA damage response (DDR) checkpoint. It has been demonstrated that DDR checkpoint proteins are activated in preneoplastic and neoplastic lesions of the skin, lung, and breast ${ }^{26-28}$; and it has been proposed that activation of the checkpoint acts as a barrier to cancer progression until mutations in checkpoint genes allow the growth of cells through the checkpoint despite DNA damage, leading to genome instability and cancer progression. Loss of heterozygosity at the FHIT locus, and most likely at the WWOX and other fragile loci, is concomitant with DDR checkpoint activation. ${ }^{1,26-28}$ We hypothesize that DNA breakage at the fragile gene loci actually may be the event that triggers the DDR and, paradoxically, loss of expression of the FHIT gene because of this breakage alters the DDR checkpoint, because Fhit is involved in normal responses to DNA damage. ${ }^{29-31}$ In addition, it recently was reported that Fhit-negative sebaceous gland carcinomas can exhibit impaired homologous recombination repair because of deletions of the BRCA1 or BRAC2 genes. ${ }^{32}$ Because basal-like breast cancers have lost or reduced Fhit protein, these tumors are likely to have defective, activated DDR checkpoints and may be especially sensitive to inhibitors of checkpoint proteins, similar to BRCA1-deficient tumors. ${ }^{16,18,33-35}$ Indeed, it has been reported that the Chk1 protein is highly expressed in triple-negative breast cancers. ${ }^{36}$

HER-2 expression was related independently to both Fhit and Wwox in our regression analysis, although the relation to Wwox did not reach significance in our univariate analysis. However, the concordant loss of Fhit and Wwox was not correlated with HER-2 expression. The relation between Fhit, Wwox, and HER-2 expression will need more detailed examination to determine whether fragile gene inactivation also contributes to the development of some HER-2 overexpressing breast tumors or whether the apparent correlation is because of complex interactions among several proteins, including Wwox and Fhit.

The other marker that had a strong and independent, inverse correlation with Fhit and Wwox expression was EGFR, which is a target for basal-like breast cancer treatment that currently is being investigated. ${ }^{17}$ Thus, it will be important to learn more regarding the correlations between EGFR and Wwox/Fhit expression in vivo and the possible effects of these correlations on responses to experimental EGFR-based therapies.

We also examined the expression of AP $2 \alpha$ and AP2 $\gamma$, proteins that interact physically with Wwox. ${ }^{10,37}$ 
Nuclear AP $2 \gamma$ expression also was more frequent in basallike tumors, perhaps partially because of Wwox reduction, which would release AP2 $\gamma$ from cytoplasmic binding to Wwox. AP2 $\gamma$ would then be free to act as a transcriptional regulator in the nucleus. However, we did not observe a significant association between nuclear AP $2 \alpha$ or AP $2 \gamma$ and Wwox expression in specific breast cancer subtypes; among the basal-like cancers, there were only 3 that strongly expressed Wwox, too few for a statistical analysis. Because there are many known and potential Wwox interactor proteins, we will need to isolate specific interactors in specific breast normal and cancer subtypes to define the hierarchy of important Wwox effector proteins. Also, the current in vivo analyses suggest that the mechanisms that control the levels of AP2 transcription factor expression are more complex than illustrated by the in vitro studies.

Recently, a role for $B R C A 1$ as stem cell regulator was described, ${ }^{38}$ and it is possible that Fhit also has a role, albeit undefined, in stem cells, as suggested recently for hematopoietic stem cells. ${ }^{39}$ Thus, it is possible that therapies based on the inhibition of a DDR checkpoint, even an abnormal checkpoint such as observed for BRCA1and/or Fhit-deficient cells, may be a targeted strategy for the treatment of basal-like/triple-negative breast cancers and even may target the stem cells of such cancers.

\section{Conflict of Interest Disclosures}

Supported by US Public Health Service/National Cancer Institute Grant CA120516, Hacettepe University Research Fund Grant 05D11101003, and the Stephanie Spielman Fund.

\section{References}

1. Durkin SG, Ragland RL, Arlt MF, et al. Replication stress induces tumor-like microdeletions in FHIT/FRA3B. Proc Natl Acad Sci USA. 2008;105:246-251.

2. Tsantoulis PK, Kotsinas A, Sfikakis PP, et al. Oncogeneinduced replication stress preferentially targets common fragile sites in preneoplastic lesions. A genome-wide study. Oncogene. 2008;27:3256-3264.

3. Thavathiru E, Ludes-Meyers JH, MacLeod MC, Aldaz CM. Expression of common chromosomal fragile site genes, WWOX/FRA16D and FHIT/FRA3B is downregulated by exposure to environmental carcinogens, UV, and BPDE but not by IR. Mol Carcinog. 2005;44:174-182.

4. Guler G, Uner A, Guler N, et al. The fragile genes, FHIT and WWOX, are coordinately inactivated in invasive breast cancer: correlations with clinical features. Cancer. 2004;100: 1605-1614.
5. Guler G, Uner A, Guler N, et al. Concordant loss of fragile genes, Fhit and Wwox, in breast cancer development. Pathol Int. 2005;55:471-478.

6. Guler G, Iliopoulos D, Guler N, et al. Wwox and Ap2 $\gamma$ expression levels predict tamoxifen response. Clin Cancer Res. 2007;13:6115-6121.

7. Nunez MI, Ludes-Meyers J, Abba MC, et al. Frequent loss of WWOX expression in breast cancer: correlation with estrogen receptor status. Breast Cancer Res Treat. 2005;89: 99-105.

8. Sbrana I, Veroni F, Nieri M, et al. Chromosomal fragile sites FRA3B and FRA16D show correlated expression and association with failure of apoptosis in lymphocytes from patients with thyroid cancer. Genes Chromosomes Cancer. 2006;45:429-436.

9. Ishii $\mathrm{H}$, Vecchione A, Furukawa $\mathrm{Y}$, et al. Expression of FRA16D/WWOX and FRA3B/FHIT genes in hematopoietic malignancies. Mol Cancer Res. 2003;1:940-947.

10. Aqeilan RI, Palamarchuk A, Weigel RJ, et al. Physical and functional interactions between the Wwox tumor suppressor protein and the AP-2gamma transcription factor. Cancer Res. 2004;64:8256-8261.

11. Aqeilan RI, Donati V, Palamarchuk A, et al. WW domaincontaining proteins, WWOX and YAP, compete for interaction with ErbB-4 and modulate its transcriptional function. Cancer Res. 2005;65:6764-6772.

12. Bosher JM, Totty NF, Hsuan JJ, et al. A family of AP-2 proteins regulates c-erbB-2 expression in mammary carcinoma. Oncogene. 1996;17:1701-1707.

13. Perou CM, Sorlie T, Eisen MB, et al. Molecular portraits of human breast tumours. Nature. 2000;406:747-752.

14. Sorlie T, Perou CM, Tibshirani R, et al. Gene expression patterns of breast carcinomas distinguish tumor subclasses with clinical implications. Proc Natl Acad Sci USA. 2001; 98:10869-10874.

15. Reis-Filho JS, Tutt ANJ. Triple negative tumours: a critical review. Histopathology. 2008;52:108-118.

16. Yehiely F, Moyano JV, Evans RE, et al. Deconstructing the molecular portrait of basal-like breast cancer. Trends $\mathrm{Mol}$ Med. 2006;12:537-544.

17. Cleator S, Heller W, Coombs RC. Triple-negative breast cancer: therapeutic options. Lancet Oncol. 2007;8:235-244.

18. Turner NC, Reis-Filho JS, Russell AM, et al. BRCA1 dysfunction in sporadic basal-like breast cancer. Oncogene. 2006;26:2126-2132.

19. Turner NC, Reis-Filho JS. Basal-like breast cancer and the BRCA1 phenotype. Oncogene. 2006;25:5846-5853.

20. Nielson TO, Hsu FD, Jensen K, et al. Immunohistochemical and clinical characterization of the basal-like subtype of invasive breast carcinoma. Clin Cancer Res. 2004;10:5367-5374.

21. Cheang MC, Voduc D, Bajdik C, et al. Basal-like breast cancer defined by 5 biomarkers has superior prognostic value than triple-negative phenotype. Clin Cancer Res. 2008;14:1368-1376. 
22. Fong LY, Fidanza V, Zanesi N, et al. Muir-Torre-like syndrome in Fhit-deficient mice. Proc Natl Acad Sci USA. 2000;97:4742-4747.

23. Zanesi N, Fidanza V, Fong LY, et al. The tumor spectrum in FHIT-deficient mice. Proc Natl Acad Sci USA. 2001; 98:10250-10255.

24. Aqeilan RI, Trapasso F, Hussain S, et al. Targeted deletion of Wwox reveals a tumor suppressor function. Proc Natl Acad Sci USA. 2007;104:3949-3954.

25. Turner BC, Ottey M, Zimonjic DB, et al. The fragile histidine triad/common chromosome fragile site $3 \mathrm{~B}$ locus and repair-deficient cancers. Cancer Res. 2002;62:4054-4060.

26. Gorgoulis VG, Vassiliou LV, Karakaidos P, et al. Activation of the DNA damage checkpoint and genomic instability in human precancerous lesions. Nature. 2005;434:907-913.

27. Bartkova J, Horejsi Z, Koed $\mathrm{K}$, et al. DNA damage response as a candidate anti-cancer barrier in early human tumorigenesis. Nature. 2005;434:864-870.

28. Bartek J, Bartkova J, Lukas J. DNA damage signalling guards against activated oncogenes and tumour progression. Oncogene. 2007;26:7773-7779.

29. Ishii $\mathrm{H}$, Mimori $\mathrm{K}$, Inoue $\mathrm{H}$, et al. Fhit modulates the DNA damage checkpoint response. Cancer Res. 2006;66:11287-11292.

30. Ishii H, Wang Y, Huebner K. A Fhit-ing role in the DNA damage checkpoint response. Cell Cycle. 2007;6:1044-1048.

31. Pichiorri F, Ishii $\mathrm{H}$, Okumura $\mathrm{H}$, et al. Molecular parameters of genome instability: roles of fragile genes at common fragile sites. J Cell Biochem. 2008;104:1525-1533.
32. Becker K, Goldberg M, Helmbold P, et al. Deletions of BRCA1/2 and p53 R248W gain-of-function mutation suggest impaired homologous recombination repair in fragile histidine triad-negative sebaceous gland carcinomas. $\mathrm{Br} J$ Dermatol. 2008;159:1282-1289.

33. Bryant HE, Schultz N, Thomas HD, et al. Specific killing of BRCA2-deficient tumours with inhibitors of poly(ADPribose) polymerase. Nature. 2005;434:913-917.

34. Farmer H, McCabe R, Lord CJ, et al. Targeting the DNA repair defect in BRCA mutant cells as a therapeutic strategy. Nature. 2005;434:917-921.

35. O'Connor MJ, Martin NMB, Smith GCM. Targeted cancer therapies based on the inhibition of DNA strand break repair. Oncogene. 2007;26:7816-7824.

36. Verlinden L, Vandem Bempt I, Eelen G, et al. The E2Fregulated gene Chk1 is highly expressed in triple-negative estrogen receptor-/progesterone receptor-/Her $1-$ breast carcinomas. Cancer Res. 2007;67:6574-6581.

37. Iliopoulos D, Guler G, Han SY, et al. Roles of FHIT and WWOX fragile genes in cancer. Cancer Lett. 2006;232:2736.

38. Liu S, Ginestier C, Charafe-Jauffret E, et al. BRCA1 regulates human mammary stem/progenitor cell fate. Proc Natl Acad Sci USA. 2008;105:1680-1685.

39. Ishii $\mathrm{H}$, Mimori $\mathrm{K}$, Ishikawa $\mathrm{K}$, et al. FHIT-deficient hematopoietic stem cells survive hydroquinone treatment long-term, carrying precancerous alterations. Cancer Res. 2008;68:3662-3670 